

\title{
COSTE ÓPTIMO Y VIABILIDAD ECONÓMICA DE LA REHABILITACIÓN ENERGÉTICA DE VIVIENDAS EN ESPAÑA
}

OPTIMAL COST AND THE ECONOMIC VIABILITY OF ENERGYEFFICIENT HOUSING RENOVATION IN SPAIN

\author{
PABLO FERNÁNDEZ ANS \\ Arquitecto Técnico e Ingeniero de Edificación \\ Jefe Técnico \\ Rehabilita Energía, A Coruña, España \\ https://orcid.org/0000-0001-7588-8153 \\ pabloans@rehabilitaenergia.com
}

\section{RESUMEN}

Las exigencias europeas de ahorro energético en edificación establecen las directrices para que cada país miembro defina su propia metodología de coste óptimo, basada en términos de coste-eficacia, tanto para obra nueva como para rehabilitación. En el caso de España, actualmente esa metodología se encuentra en fase de desarrollo, por lo que el presente estudio aplica las directrices europeas y plantea diferentes medidas de rehabilitación pasivas en la envolvente para dos tipologías de edificios representativos en dicho país, un edificio entre medianeras en casco histórico y otro bloque de viviendas aislado, situados en cinco zonas climáticas diferentes. El trabajo relaciona la demanda y consumo energético ( $\mathrm{kW} \bullet \mathrm{h} / \mathrm{m} 2$ año) con el coste global $(€)$ para diferentes propuestas, obteniendo

los valores de coste óptimo y períodos de amortización. Se propone, además, el indicador de "coste 30", como el coste adecuado para conseguir amortizaciones inferiores a 30 años, y se amplía el análisis incorporando el salario mínimo interprofesional a la inversión. Los resultados concluyen que la metodología de coste óptimo permite obtener valores adecuados y que, en ese marco, existe un abanico de intervenciones válidas que dependen principalmente de la tipología, la zona climática y los costes de inversión.

Palabras clave

desempeño térmico, renovación arquitectónica, ahorro de energía, costes de construcción.

\section{ABSTRACT}

The European requirements for energy savings in buildings set the guidelines by which each member country establishes their own optimal cost methodology with respect to cost-effectiveness, both in new buildings and renovations. In the case of Spain, this methodology is currently in the development phase. Therefore, this study applies the European guidelines and proposes different passive renovation measures in envelopes for representative building typologies in Spain: a building between party walls in a historic district and an apartment building, located in five different climatic zones. The study relates energy consumption and demand $(\mathrm{kW} \bullet \mathrm{h} / \mathrm{m} 2$ year) and global cost $(€)$ for different proposals, and determines optimal cost values and amortization periods. In addition, it proposes the cost30 indicator as the appropriate cost that enables amortization periods of less than 30 years; furthermore, the analysis is broadened by considering minimum wage in the investment. The results conclude that suitable values may be obtained with the optimal cost methodology, and that there are a variety of different valid renovation measures that depend mainly on typology, climatic zone and investment costs. 


\section{INTRODUCCIÓN}

Según las recomendaciones de la Comunidad Europea, actualmente es crucial reducir el consumo de energía en todos los sectores, siendo el sector de la edificación responsable del $40 \%$ total de dicho consumo. Las bases para esta reducción se establecen en la Directiva 2010/31/EU (Unión Europea, 2010), que desarrolla nuevos planteamientos y exigencias en el área de la eficiencia energética en la edificación y que han sido traspuestas a la normativa de España (Código Técnico de la Edificación, 2017).

Por lo que respecta a la rehabilitación de edificios, según estimaciones de la Unión Europea (2019), el 35\% de los edificios tienen más de 50 años y el $75 \%$ del stock es ineficiente, con un porcentaje de renovación inferior al $1,2 \%$, de modo que la rehabilitación presenta un gran potencial de mejora energética, que puede suponer reducir consumos y emisiones de $\mathrm{CO}_{2}$ en torno a un $5 \%$.

Las dos grandes líneas para abordar el problema energético de la edificación en Europa son la apuesta hacia estándares de edificios de consumo de energía casi nulo (Nearly zero-energy buildings, nZEB) y a la rehabilitación. En este último caso, el principal problema es la limitación física de un edificio ya existente, y las medidas a adoptar diferirán a las planteadas en edificios de nueva construcción, por lo que las soluciones deberán ser "técnica, funcional y económicamente viables", según recoge el artículo 7 de la Directiva (Unión Europea, 2010).

Pero en la ecuación energética es necesario incluir el parámetro económico que es clave para la viabilidad económica de las intervenciones, obligando a definir de entre todas las posibles opciones aquellas más adecuadas para alcanzar un equilibrio óptimo entre las inversiones realizadas y los costes energéticos ahorrados hasta su depreciación.

Por ello, la Comunidad Europea ha establecido la necesidad de que cada Estado miembro defina su propio marco metodológico (en fase de desarrollo en la mayoría de los países) que permita calcular y comparar los óptimos de rentabilidad, establecidos en el Reglamento Delegado RD 244/2012 y en sus directrices aclaratorias (Unión Europea, 2012b).

La metodología a aplicar deberá ser particularizada y dependerá de cada Estado miembro:

"Despite a common methodology to calculate cost optimal levels, the results are not fully comparable between countries, as Member States are free, for example, to choose the macroeconomic or financial perspective when calculating cost optimal values or have different national rules to calculate energy performance of buildings" (ECOFYS y EURIMA, 2015).
Con una metodología todavía sin aprobar en España acorde al RD 244/2012 y al estándar UNE-EN 15459:2008 (AENOR-CEN, 2008), la única referencia oficial (Ministerio de Fomento, 2013) establece un análisis comparativo de diferentes medidas y paquetes de medidas en edificios existentes y de nueva construcción, para diferentes zonas climáticas. Dada la ausencia actual de regulación y unas crecientes exigencias energéticas en la normativa, hacia estándares de NZEB, se considera el análisis del coste óptimo como un aspecto clave sobre el que se plantea el presente estudio.

Existen otros trabajos que ya han abordado el tema y que analizan un amplio espectro de edificios, entre ellos destacan: el Episcope Project (2016); el proyecto Concerted Action (CA-EPBD, 2016) o las guías publicadas por ECOFYS en colaboración con la European Insulation Manufacturers Association (EURIMA) (2015); el proyecto TABULA (2012), que establece criterios comunes de clasificación del stock de edificios de acuerdo con la edad, tamaño y zona climática, además de otros parámetros energéticos.

Bajo la misma metodología, otros estudios analizan un amplio espectro de tipologías de edificios, para climas representativos de Europa e, incluso, consideran edificios de consumo casi nulo NZEB (Zangheri, Armani, Pietrobon y Pagliano, 2018), en Italia (Corrado, Ballarini y Paduos, 2014) o incluyendo otros usos como oficinas (Arumägi, Simson, Kuusk, Kalamees y Kurnitski, 2017) o educacional (Niemelä, Kosonen y Jokisalo, 2016).

Algunos autores plantean diferentes niveles definiendo "rehabilitaciones leves, shallow renovations" para intervenciones que alcanzan ahorros energéticos del $32 \%$, - "rehabilitaciones intensas, deep renovations", que llegan al 80\% (ECOFYS y EURIMA, 2012), denominadas en otras investigaciones como "rehabilitaciones básicas" o "plus" (Pérez, Calama y Flores, 2016).

También se han incorporado aspectos sociales que valoran el coste de inversión por familia (De la Cruz, De la Cruz y Simón, 2018) en niveles de inversiones leves, moderadas o intensas, dependiendo del coste en $€$ /vivienda (RePrograma, 2015), (Luxán, 2017).

No existe una metodología oficial de coste óptimo que aporte una base de datos representativa de edificios de viviendas en diferentes climas de España, ni tampoco una valoración de la repercusión económica a las familias. A partir de esas carencias se formula el estudio actual, para intervenciones pasivas en la envolvente, con el objetivo de valorar el coste y amortización de diferentes propuestas de rehabilitación energética en cinco zonas climáticas de España, en la Comunidad Autónoma de Andalucía, cuantificando los parámetros fundamentales de demanda energética conjunta de calefacción y refrigeración $(\mathrm{kW} \bullet \mathrm{h} /$ m2 año) e inversión económica ( $€$ ). 


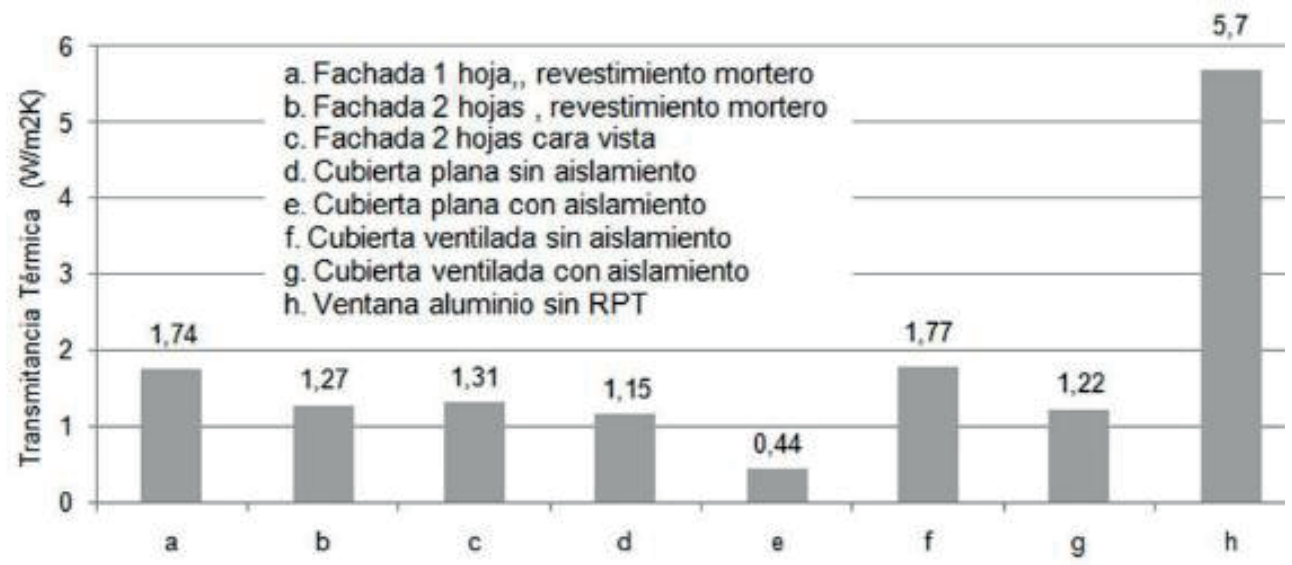

Figura 1. Valores de transmitancia térmica $U(\mathrm{~W} / \mathrm{m} 2 \mathrm{~K})$ en los edificios de referencia. Fuente: Elaboración del autor

Para conseguir dicho fin, se analizan dos tipologías de edificios residenciales representativos de una gran parte del parque edificado. Uno de ellos es un edificio entre medianeras, situado en un área de casco histórico, y el segundo, un edificio en bloque, ubicado en zona nueva residencial.

Forma parte del objetivo seguir las directrices de coste óptimo, priorizando intervenciones pasivas de acuerdo con el espíritu global de la Directiva 2010/31/EU, e incorporar la capacidad financiera de las familias incluyendo el salario mínimo interprofesional.

\section{METODOLOGÍA}

La metodología empleada en el estudio se indica a continuación:

- Definición de dos edificios de referencia, un edificio entre medianeras (EM) y un edificio en bloque (EB), así como sus condiciones constructivas y geométricas, representativas de gran parte del parque edificado en España, en base a datos estadísticos.

- Definición de cinco zonas climáticas de estudio, de acuerdo con el CTE (2017).

- Definición de las intervenciones pasivas a realizar sobre la envolvente, agrupadas en medidas/paquetes/ variantes, según las directrices de la metodología de coste óptimo del RD 244/2012.

- Cálculo energético, mediante uso de la aplicación de simulación dinámica Herramienta Unificada LIDERCALENER (HULC) (2017), herramienta reconocida en la normativa española, para obtener valores de demanda energética conjunta de calefacción y refrigeración, y consumos de energía primaria $\left(\mathrm{kW} \bullet \mathrm{h} / \mathrm{m}^{2}\right.$ año).
- Cálculo económico, a través del cual se obtiene el coste global y el valor residual de cada medida/paquete/ variante. El coste global se compone de la inversión inicial según precios de mercado y bases de datos de la construcción, más el coste de la energía durante la vida útil. Se amplía el estudio valorando la repercusión económica por familia respecto al salario mínimo interprofesional (SMI).

- Representación gráfica de coste óptimoy amortizaciones. Se amplía el estudio con la propuesta de "coste30", para lograr amortizaciones inferiores a 30 años.

\section{DEFINICIÓN DE LOS EDIFICIOS DE REFERENCIA}

Para abarcar el ámbito del estudio se han definido dos edificios de referencia del parque edificado, de acuerdo a publicaciones estadísticas, lo que permitirá ampliar el área de conocimiento y realizar un análisis comparativo entre ambos.

Respecto a la superficie, en Andalucía la mayor parte de las viviendas $(29,51 \%)$ tiene una superficie comprendida entre

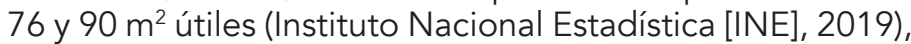
con un gran porcentaje de fachadas de ladrillo $(54,35 \%)$ con revestimientos de mortero $(34,99 \%)$, cubierta plana transitable y carpinterías exteriores de aluminio $(86,25 \%)$, (Ministerio de Fomento, 2018).

Con esa caracterización constructiva se han definido los elementos de la envolvente de los casos de referencia, cuyos valores de transmitancia térmica resultantes se exponen en la Figura 1.

Considerando los parámetros anteriores, se han determinado dos edificios de referencia reales, un edificio entre medianeras (EM) y un edificio en bloque (EB) (Figura 2), cuyas características se resumen en la Tabla 1. 


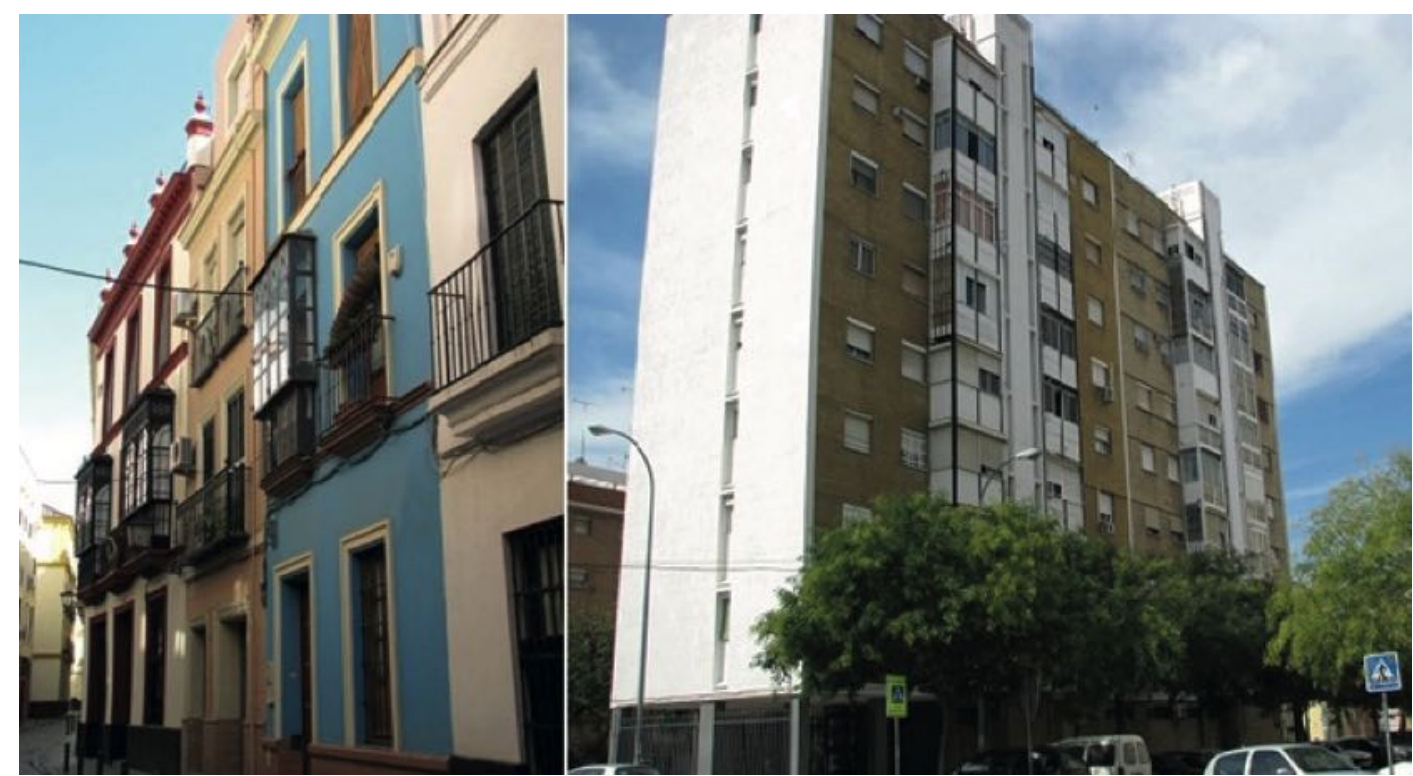

Figura 2. Edificios de referencia: edificio medianeras (EM) y edificio bloque (EB). Fuente: Fotografías del autor.

\begin{tabular}{|c|c|c|c|c|}
\hline & $\begin{array}{l}\text { Descripción } \\
\text { edificio }\end{array}$ & $\begin{array}{l}\text { Geometría } \\
\text { (Sup. útil } / \mathrm{n}^{\circ} \\
\text { viviendas) }\end{array}$ & $\begin{array}{l}\text { Ventanas } \\
\left(m^{2} / \%\right)\end{array}$ & $\begin{array}{c}\text { Características constructivas } \\
\mathbf{U}\left(\mathrm{W} / \mathrm{m}^{2} \mathrm{~K}\right)\end{array}$ \\
\hline $\begin{array}{l}\text { Edificio } \\
\text { medianeras } \\
(\text { (EM) }\end{array}$ & $\begin{array}{c}\text { Años: } 1900-1920 \\
\text { Solar: } 180 \mathrm{~m}^{2} \\
\text { Vol.: } 2.250 \mathrm{~m}^{3} \\
\text { Sup. fachadas: } \\
111,56 \mathrm{~m}^{2} \\
\text { Sup. construida: } 450 \mathrm{~m}^{2}\end{array}$ & $100 \mathrm{~m}^{2} / 2$ & $\begin{array}{c}18,54 \mathrm{~m}^{2} / \\
2,56 \%\end{array}$ & $\begin{array}{l}\text { Muro ladrillo, } 1 \text { hoja: } U=1,74 \\
\text { Medianeras: } U=2,33 \\
\text { Cubierta plana "andaluza": } U=1,15 \\
\text { Ventana aluminio, vidrio sencillo. } \\
U=5,70, \mathrm{~g} \text { (factor solar) }=0,85\end{array}$ \\
\hline $\begin{array}{c}\text { Edificio } \\
\text { bloque (EB) }\end{array}$ & $\begin{array}{c}\text { Años: } 1961-1980 \\
\text { Solar: } 392,95 \mathrm{~m}^{2} \\
\text { Vol.: } 19.254,55 \mathrm{~m}^{3} \\
\text { Sup. fachadas: } \\
2.732,80 \mathrm{~m}^{2} \\
\text { Sup. construida: } \\
2.841 \mathrm{~m}^{2}\end{array}$ & $90 \mathrm{~m}^{2} / 20$ & $\begin{array}{c}771,20 \mathrm{~m}^{2} / \\
18,60 \%\end{array}$ & $\begin{array}{c}\text { Muro ladrillo visto, } 2 \text { hojas: } U=1,31 \\
\text { Cubierta plana "catalana": } U=1,22 \\
\text { Ventana aluminio, vidrio sencillo } \\
U=5,70, g \text { (factor solar) }=0,85\end{array}$ \\
\hline
\end{tabular}

Tabla 1. Características de los edificios de referencia. Fuente: Elaboración del autor.

- Edificio entre medianeras (EM): situado en casco histórico, tres plantas, una fachada exterior y un patio interior.

- Edificio en bloque (EB): residencial de viviendas, aislado, doce plantas y cuatro fachadas exteriores.

\section{DEFINICIÓN DE LAS ZONAS CLIMÁTICAS}

En España se definen quince zonas climáticas en el CTE (2017) abarcando un amplio espectro de áreas frías y cálidas y que han generado en la arquitectura tradicional diferentes soluciones y estrategias bioclimáticas pasivas en su adaptación al medio, desde el uso de cubiertas vegetales (Molina y Fernández-Ans, 2013) a estrategias de ventilación natural también utilizadas en climas templados continentales (Mercado, Esteves, Barea y Filippín, 2018).

Los casos de estudio se establecen para la Comunidad de Andalucía (España), que comprende siete tipos de clima representados en la Figura 3; los cuales, a su vez, corresponden a cinco zonas climáticas, de acuerdo con la zonificación climática establecida por la normativa (CTE, 2017, apéndice B), por lo que el estudio es extrapolable a otras provincias.

La determinación de zonas climáticas se define mediante una letra, correspondiente a la división de invierno, y un número, correspondiente a la división de verano, según la siguiente clasificación: 


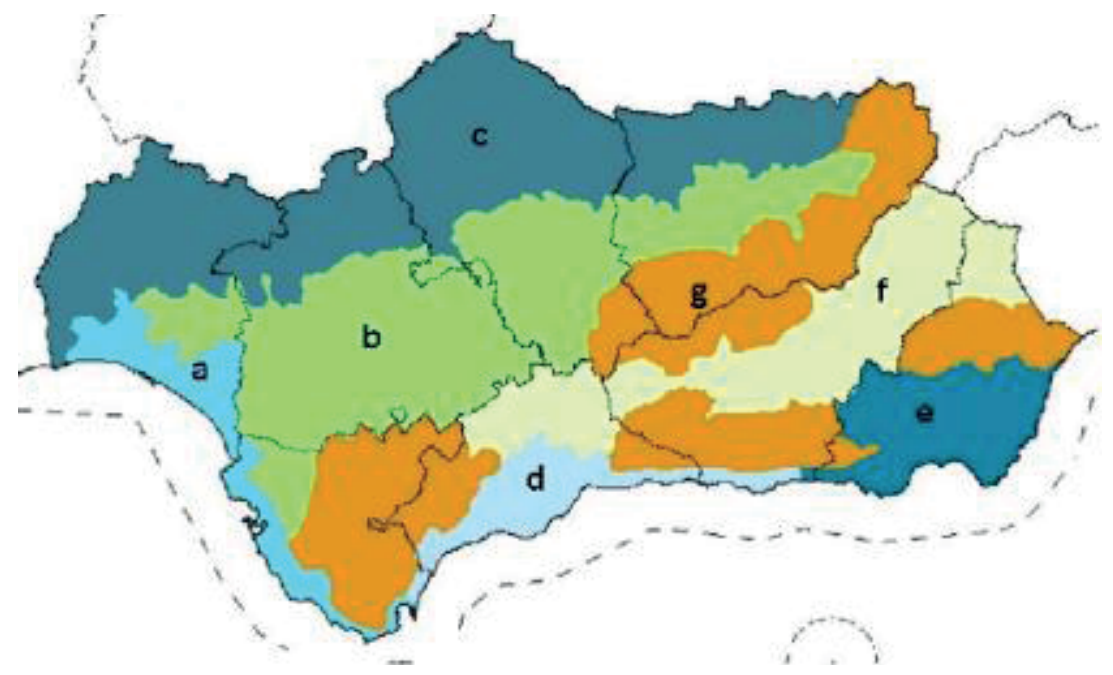

Figura 3. Tipos de clima mediterráneo en Andalucía: Oceánico (a), Continental (b), Semiárido (c), Subtropical (d), Subdesértico (e), Continental (f), De montaña (g). Fuente: Consejería de Medio Ambiente (2019).

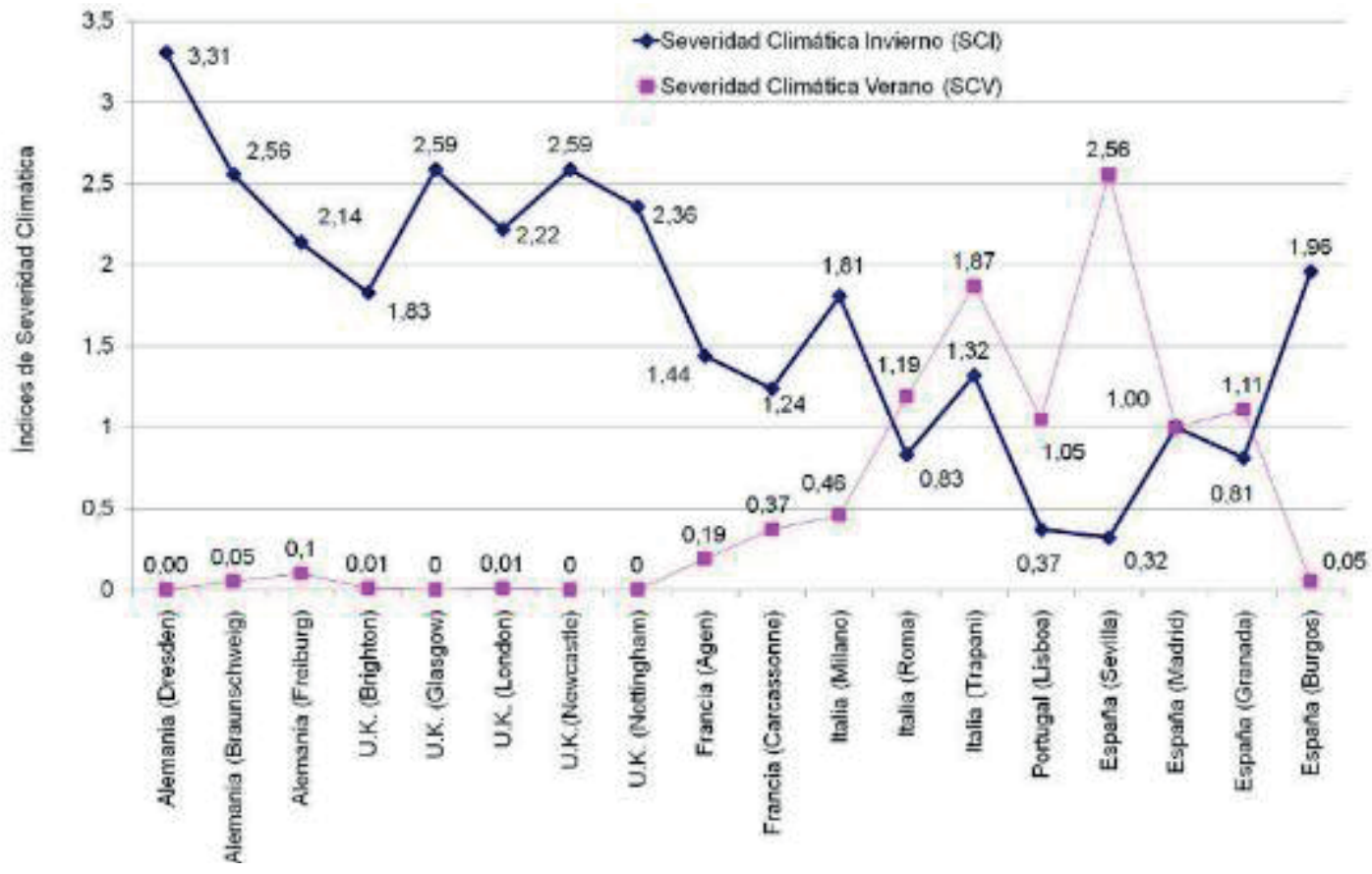

Figura 4. Índices de Severidad Climática en Europa. Fuente: Elaboración del autor. Fuente: Passive-On Project (European Commission, 2007).

- Zona climática A3: Cádiz-Málaga

- Zona climática A4: Almería- Huelva

- Zona climática B4: Córdoba- Sevilla

- Zona climática C3: Granada

- Zona climática C4: Jaén

En comparación con otras ciudades europeas, algunas zonas presentan altos índices de severidad climática en verano $(\mathrm{SCV})$ e intermedios en invierno $(\mathrm{SCl})$, tal como se expone en la Figura 4.

\section{DEFINICIÓN DE INTERVENCIONES PASIVAS}

Se determinan, en este punto, diferentes intervenciones pasivas de rehabilitación que ofrecen una reducción de la demanda energética del edificio, agrupadas en: medidas, paquetes y variantes, en consonancia con la Directiva 2010/31/EU y otros estudios (Suárez y Fragoso, 2016).

Las medidas son intervenciones en fachadas y cubiertas, por el interior y por el exterior (SATE, Sistema Aislamiento Térmico Exterior) así como la sustitución de ventanas. En 


\begin{tabular}{|c|c|c|c|}
\hline & Medidas & Variante 1: Paquetes individuales & $\begin{array}{l}\text { Variante 2: } \\
\text { Intervención } \\
\text { total }\end{array}$ \\
\hline Fachada exterior & $\begin{array}{l}5 \mathrm{~cm} \text { EPS }(U=0,52) \\
5 \mathrm{~cm} \mathrm{PIR}(U=0,42)\end{array}$ & \multirow{6}{*}{$\begin{array}{c}\text { Fachada exterior (EPS) } \\
\text { Fachada interior (XPS) } \\
\text { Cubierta exterior (BA) } \\
\text { Cubierta interior (MW) } \\
\text { Ventana aluminio, vidrio bajo e } \\
\text { Ventana PVC, vidrio bajo e }\end{array}$} & \multirow{6}{*}{$\begin{array}{c}\text { Fachada exterior } \\
\text { (EPS) } \\
+ \\
\text { Cubierta exterior } \\
\text { (BA) } \\
+ \\
\text { Ventana PVC, } \\
\text { vidrio bajo e }\end{array}$} \\
\hline \multirow[b]{2}{*}{ Cubierta exterior } & $\begin{array}{l}5 \mathrm{~cm} \text { EPS }(U=0,50) \\
5 \mathrm{~cm} \text { XPS }(U=0,47) \\
5 \mathrm{~cm} \mathrm{MW}(U=0,53) \\
5 \mathrm{~cm} \text { TER }(U=0,23) \\
5 \mathrm{~cm} \text { PUR }(U=0,48)\end{array}$ & & \\
\hline & $\begin{array}{l}7 \mathrm{~cm} \mathrm{XPS}(U=0,34) \\
7 \mathrm{~cm} \mathrm{BA}(U=0,47)\end{array}$ & & \\
\hline Cubierta interior & $\begin{array}{l}7 \mathrm{~cm} \text { EPS }(U=0,35) \\
7 \mathrm{~cm} \mathrm{MW}(U=0,37)\end{array}$ & & \\
\hline Carpinterías aluminio & $\begin{array}{c}\text { Al + vidrio }(U=2,92) \\
\text { Al + vidrio bajo e }(U=1,84)\end{array}$ & & \\
\hline Carpinterías PVC & $\begin{array}{c}\text { PVC + vidrio }(U=2,74) \\
\text { PVC + vidrio bajo e }(U=1,66)\end{array}$ & & \\
\hline
\end{tabular}

Tabla 2. Medidas/paquetes/variantes adoptadas. Fuente: Elaboración del autor.

\begin{tabular}{|c|c|}
\hline Temperatura consigna $\left({ }^{\circ} \mathrm{C}\right)$ & Verano: $25-27{ }^{\circ} \mathrm{C}$. Invierno: $17-20^{\circ} \mathrm{C}$ \\
\hline Ventilación & Verano: 4/ren/hora por la noche (1-8h) \\
\hline Infiltraciones & 0,24 ren/hora para bloques de viviendas \\
\hline Huecos & Factor de sombra 0,7 ; persianas bajadas $30 \%$. \\
\hline Demanda ACS / vivienda & 56 litros/día a $60^{\circ} \mathrm{C}$ \\
\hline Climatización & $\begin{array}{c}\text { 60\% viviendas: Bomba calor aire/aire 2x1 (EER 2,5; COP 2,7) } \\
40 \% \text { viviendas: Bomba calor aire/aire } 1 \times 1 \text { (EER 2,5; COP 2,7) + calefactor eléctrico } \\
2 \mathrm{~kW} \text { térmicos (Efecto Joule) }\end{array}$ \\
\hline
\end{tabular}

Tabla 3. Principales parámetros de cálculo considerados en HULC. Fuente: Elaboración del autor.

todos los casos se han cumplido los valores límites de transmitancia térmica (CTE, 2017).

Los paquetes definen las soluciones constructivas empleando diversos aislantes (EPS, expanded polystyrene; PIR, Polyisocyanurate; XPS, extruded polystyrene; PUR, Polyurethane foam; MW, mineral wool).

Las variantes agrupan diferentes medidas y paquetes, ofreciendo varias opciones de rehabilitación energética.

En cuanto a los espesores de aislamientos, en fachadas se definen de $5 \mathrm{~cm}$ y en cubiertas, de $7 \mathrm{~cm}$, compuestas por baldosas de hormigón con aislamiento XPS incorporado (BA, baldosas aislantes). Las carpinterías exteriores son de aluminio con rotura de puente térmico o PVC, en ambos casos con vidrios aislantes y bajo emisivos.
Finalmente, se ha analizado una Variante 1, compuesta de varias medidas individuales, y otra Variante 2 como intervención total (Tabla 2).

\section{CÁLCULO ENERGÉTICO}

El cálculo se ha realizado con la aplicación de simulación dinámica HULC que permite establecer las demandas y consumos de energía primaria necesarios para mantener unas condiciones de confort predefinidas, según condiciones operacionales de temperatura de consigna, ocupación, iluminación y ventilación indicadas en el Apéndice $C$, perfiles de uso residencial (CTE, 2017, apéndice C).

Los principales parámetros de cálculo se indican en la Tabla 3 ; se ha considerado que un $60 \%$ de las viviendas posee 


\begin{tabular}{|c|c|c|c|c|c|c|c|}
\hline & \multirow{2}{*}{\multicolumn{2}{|c|}{ A3 }} & \multicolumn{5}{|c|}{$\begin{array}{c}\text { Zonas climáticas } \\
\text { demanda / consum॰ ( } \mathrm{WW} / \mathrm{h} / \mathrm{m}^{2} \text { año) }\end{array}$} \\
\hline & & & A4 & B4 & C3 & C4 & \\
\hline \multicolumn{8}{|c|}{ Variante 1: Paquetes individuales } \\
\hline \multirow{4}{*}{ Muros } & \multirow{2}{*}{ EM } & Fachada exterior-EPS & $28,37 / 65,2$ & $38,89 / 71,9$ & $56,18 / 91,6$ & $78,72 / 128,3$ & $73,83 / 117,4$ \\
\hline & & Fachada interior-XPS & $32,92 / 74,5$ & $40,22 / 76,1$ & $58,06 / 93,4$ & $80,58 / 137,7$ & $76,08 / 114,9$ \\
\hline & \multirow{2}{*}{ EB } & Fachada exterior-EPS & $40,27 / 91,8$ & $51,72 / 97,7$ & $72,88 / 120,9$ & $113,03 / 192,1$ & $100,61 / 151,9$ \\
\hline & & Fachada interior-XPS & $42,01 / 94,94$ & $53,55 / 102,8$ & $75,79 / 125,8$ & $115,95 / 197,1$ & $101,99 / 164,2$ \\
\hline \multirow{4}{*}{ Cubiertas } & \multirow{2}{*}{ EM } & Cubierta exterior-BA & $35,62 / 80,6$ & $43,86 / 84,49$ & $61,85 / 110,5$ & $88,60 / 151,4$ & $82,07 / 132,3$ \\
\hline & & Cubierta interior-MW & $35,24 / 79,8$ & $43,32 / 83,4$ & $61,04 / 109,1$ & $87,54 / 149,6$ & $81,09 / 130,7$ \\
\hline & \multirow{2}{*}{ EB } & Cubierta exterior-BA & $47,33 / 107,1$ & $59,39 / 114,4$ & $83,76 / 149,1$ & $130,14 / 222,4$ & $114,87 / 185,2$ \\
\hline & & Cubierta interior-MW & $47,13 / 106,7$ & $59,15 / 113,9$ & $83,44 / 148,5$ & $129,67 / 221,6$ & $114,45 / 184,5$ \\
\hline \multirow{4}{*}{ Ventanas } & \multirow{2}{*}{ EM } & Ventana alum., bajo e & $27,79 / 62,9$ & $33,41 / 64,4$ & $47,93 / 85,6$ & $66,33 / 113,3$ & $63,13 / 101,8$ \\
\hline & & Ventana PVC, bajo e & $30,16 / 68,3$ & $36,69 / 70,7$ & $51,47 / 91,9$ & $71,03 / 121,4$ & $67,71 / 109,1$ \\
\hline & \multirow{2}{*}{ EB } & Ventana alum., bajo e & $41,19 / 93,1$ & $52,45 / 100,7$ & $74,04 / 131,8$ & $112,51 / 191,3$ & $100,02 / 161,2$ \\
\hline & & Ventana PVC, bajo e & $40,80 / 92,2$ & $52,02 / 99,8$ & $73,42 / 130,7$ & $111,31 / 189,2$ & $99,14 / 159,8$ \\
\hline \multicolumn{8}{|c|}{ Variante 2: Intervención total } \\
\hline $\begin{aligned} & \text { Muro } \\
&+ \text { Cubierta } \\
&+ \text { Ventana } \\
&\end{aligned}$ & EM & $\begin{array}{l}\text { Fachada exterior-EPS } \\
\text { Cubierta exterior-BA } \\
\text { Ventana PVC, bajo e }\end{array}$ & $28,37 / 63,8$ & $34,63 / 57,1$ & $45,22 / 71,4$ & $54,79 / 83,82$ & $63,66 / 94,6$ \\
\hline $\begin{aligned} & \text { Muro } \\
&+ \text { Cubierta } \\
&+ \text { Ventana } \\
&\end{aligned}$ & EB & $\begin{array}{l}\text { Fachada exterior-EPS } \\
\text { Cubierta exterior-BA } \\
\text { Ventana PVC, bajo e }\end{array}$ & $36,18 / 81,4$ & $46,29 / 76,4$ & $63,43 / 100,2$ & $89,48 / 136,9$ & $100,41 / 148,6$ \\
\hline
\end{tabular}

Tabla 4. Demanda conjunta / Consumos (kW•h/m2 año). Edificio medianeras (EM), edificio bloque (EB).Fuente: Elaboración del autor.

un sistema multizona 2x1 de expansión directa aire/aire, y el $40 \%$ restante, un sistema compacto $1 \times 1$, incluyendo un apoyo de calefactores eléctricos.

En la Tabla 4 se presentan resultados de demanda conjunta de calefacción y refrigeración, y de consumos ( $\mathrm{kW} \bullet \mathrm{h} /$ $\mathrm{m}^{2}$ año), para las diferentes variantes y las cinco zonas climáticas.

\section{CÁLCULO ECONÓMICO}

\section{COSTE GLOBAL}

Para cada propuesta se calcula un coste global, suma del coste de inversión, funcionamiento y sustitución, así como el coste de eliminación, de acuerdo con la metodología europea (Unión Europea, 2012a) (Figura 5).

En los costes de inversión inicial se contemplan los trabajos de demolición y preparación previos, los materiales y la instalación (mano de obra, herramientas, andamios, contenedores de escombro), considerando los derivados del diseño despreciables. Se han tomado como referencia precios de fabricantes (DANOSA, s/f; URSA, s/f) y bases de precios de construcción en España (ATAYO, s/f); COAATGU, 2018; Junta de Andalucía, 2017).

El coste anual recoge los debidos al funcionamiento en un período de 30 años, asociados principalmente a los costes de la energía. Los costes de sustitución y mantenimiento se consideran nulos.

El coste de la energía se cuantifica con un mix $59,10 \%$ eléctrico $(0,12 € / \mathrm{kW} \cdot \mathrm{h})$ y $40,90 \%$ de otras fuentes $(0,035 € /$ $\mathrm{kW} \cdot \mathrm{h})$, según los factores de paso de energía primaria oficiales (Gobierno de España, 2016) y el precio del kW·h (Instituto para la Diversificación y Ahorro de la Energía [IDEA], 2016). El incremento anual de coste de la energía se ha estimado en el $4 \%$.

\section{VALOR RESIDUAL}

Es necesario considerar el valor residual a 30 años de las intervenciones, descontándolo del coste de inversión inicial según la depreciación lineal definida en el RD 244/201 (Figura 6). 


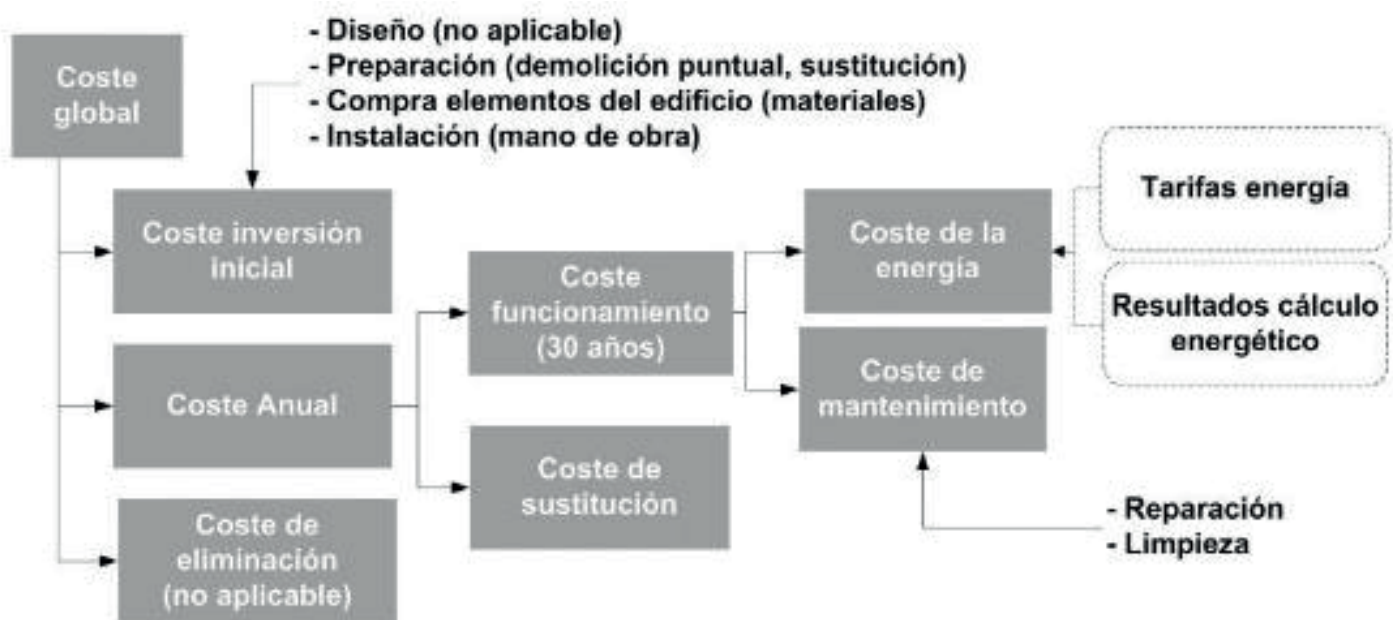

Figura 5. Categorización de costes según el marco europeo. Fuente: Directrices RD 244/2012 (Unión Europea, 2012a), Elaboración del autor.

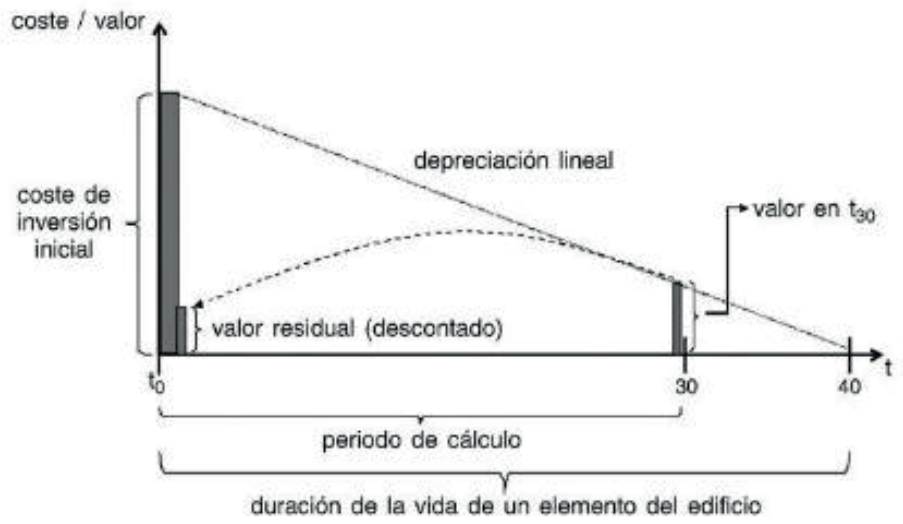

Figura 6. Depreciación del elemento de un edificio. Fuente: Directrices RD 244/2012 (Unión Europea, 2012a), Elaboración del autor.

Considerando una depreciación máxima del 100\% a 40 años, a 30 años se obtiene un valor residual del 25\%, límite asociado a la vida útil de fachadas; los resultados respectivos se indican en las Figuras 7 y 8 para la Variante 1 (6 medidas individuales) y la Variante 2 (intervención total), en las dos tipologías de edificios analizadas.

\section{RESULTADOS ECONÓMICOS}

A partir de los resultados anteriores, resulta el valor de coste global en 30 años (inversión inicial + consumo con $4 \%$ incremento precio energía - valor residual) en $€ / m 2$ de superficie construida, indicado en la Tabla 5; los costes de inversión inicial se valoran en $\mathrm{m}^{2}$ de cada solución constructiva (fachada, cubierta o ventana).

Se incorpora el aspecto social, valorando la capacidad económica para abordar el coste de la inversión inicial tanto por vivienda como por unidad familiar, en base al salario mínimo interprofesional (SMI), establecido en España en 900

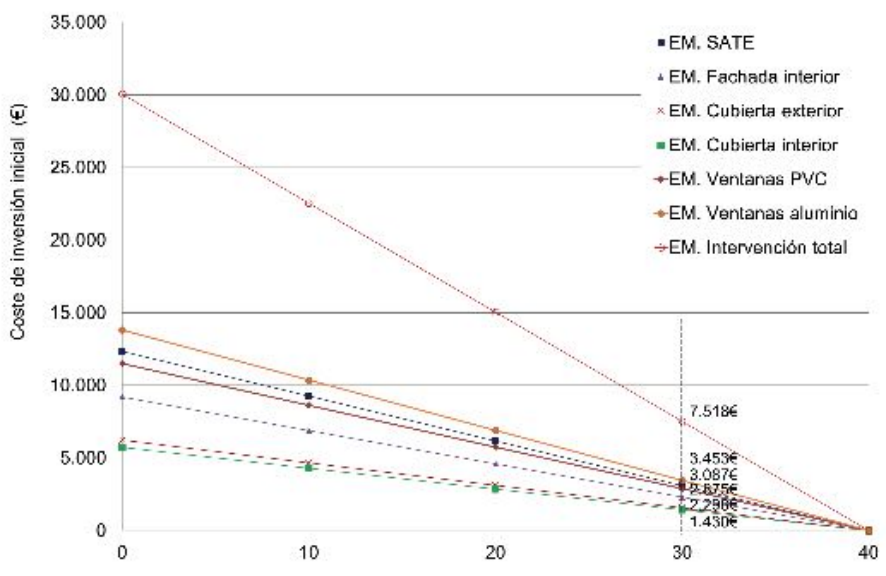

Depreciación (años) de los elementos del edificio.Edificio entre Medianeras (EM)

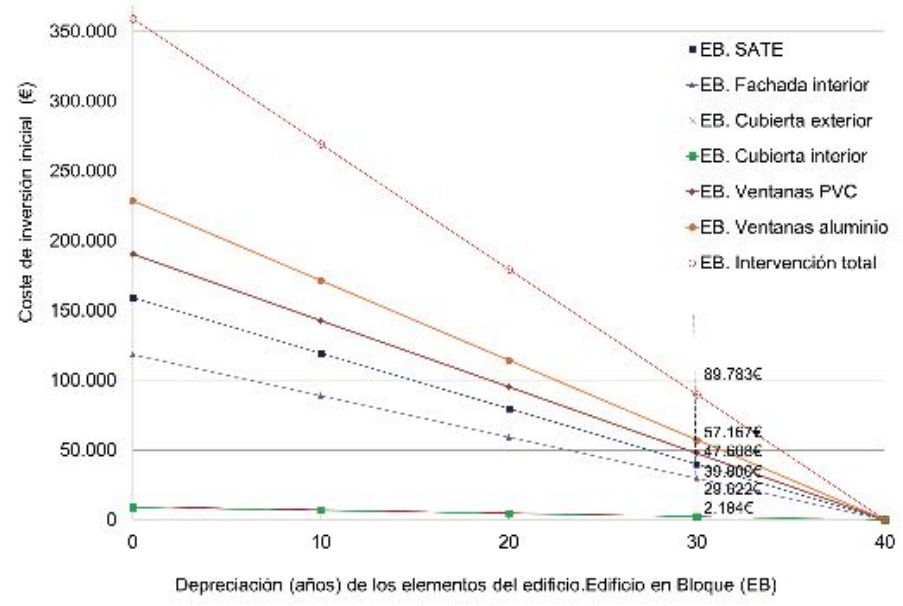

Figura 7. Depreciación y valor residual a 30 años. Edificio medianeras (EM).Fuente: Elaboración del autor.

Figura 8. Depreciación y valor residual a 30 años. Edificio en bloque (EB). Fuente: Elaboración del autor. 


\begin{tabular}{|c|c|c|c|c|c|c|c|c|c|}
\hline & \multicolumn{2}{|c|}{$\begin{array}{c}\text { Coste Global } € / \mathrm{m}^{2} \\
\text { construido }\end{array}$} & \multirow{2}{*}{$\begin{array}{l}\text { Inversión inicial } \\
€ / m^{2} \text { solución } \\
\text { constructiva }\end{array}$} & \multicolumn{2}{|c|}{$\begin{array}{c}\text { Repercusión } € / \\
m^{2} \text { vivienda }\end{array}$} & \multicolumn{2}{|c|}{ Coste $€ /$ familia } & \multicolumn{2}{|c|}{$\begin{array}{c}\mathbf{N}^{\circ} \text { veces } \\
\text { SMI }\end{array}$} \\
\hline & EM & EB & & EM & EB & $\begin{array}{c}\text { EM } \\
(2 \text { fam.) }\end{array}$ & $\begin{array}{c}\text { EB } \\
(20 \text { fam. })\end{array}$ & EM & EB \\
\hline \multicolumn{10}{|c|}{ Variante 1. Paquetes individuales } \\
\hline Fachada ext. - EPS & 48,95 & 149,12 & 48,92 & 27,44 & 56,04 & 6.170 & 7.960 & 9 & 11 \\
\hline Fachada int.- XPS & 48,24 & 143,00 & 36,41 & 20,42 & 41,71 & 4.600 & 5.925 & 7 & 8 \\
\hline Cubierta ext. - BA & 45,99 & 128,37 & 41,48 & 13,83 & 3,34 & 3.110 & 470 & 4 & 1 \\
\hline Cubierta int. - MW & 44,78 & 127,64 & 38,14 & 12,71 & 3,07 & 2.860 & 440 & 4 & 1 \\
\hline Ventana Al & 50,81 & 169,91 & 296,51 & 30,69 & 80,50 & 6.910 & 11.430 & 10 & 16 \\
\hline Ventana PVC & 49,33 & 158,78 & 246,93 & 25,56 & 67,04 & 5.750 & 9.520 & 8 & 14 \\
\hline \multicolumn{10}{|c|}{ Variante 2. Intervención total } \\
\hline $\begin{array}{l}\text { Fachada ext. EPS } \\
\text { +Cubierta ext. BA } \\
\text { +Ventana PVC }\end{array}$ & 78,49 & 191,03 & - & 66,83 & 126,43 & 15.040 & 17.960 & 21 & 26 \\
\hline
\end{tabular}

Tabla 5. Coste Global e inversión por familia. Edificio medianeras (EM), edificio bloque (EB). Fuente: Elaboración del autor.

$€ /$ mes para el año 2019 y contemplando a dos familias en el edificio entre medianeras y veinte familias en el edificio en bloque.

Los resultados indican que las intervenciones en el edificio entre medianeras suponen entre 4 y 21 veces el SMI, y de 1 a 26 para el edificio en bloque. Los valores menores se corresponden a intervenciones en cubiertas y los mayores para la Variante 2 de intervención total.

El coste por unidad familiar permite definir niveles de intervención acorde a otras publicaciones, que las delimitan en inversiones leves ( $<2.500 € /$ vivienda), moderadas (2.500-4.500 €/vivienda) o intensas ( $>4.500 € /$ /vivienda), (Re-Programa, 2015). Estudios similares establecen para la Región Metropolitana de Chile tres niveles de intervención según los ingresos familiares (Low-Medium-High incomes), con porcentajes muy bajos de inversión inicial por parte de las familias $2 \%-3 \%-0 \%$, que son financiados con ayudas del Gobierno y préstamos bancarios (García y Croxford, 2015); y otros autores consideran soluciones de bajo coste para inversiones inferiores a 4.200 €/familia (Luxán, 2017).

\section{COSTE ÓPTIMO Y AMORTIZACIÓN}

Los valores obtenidos de coste global a 30 años se relacionan con los consumos anuales $\left(\mathrm{kW} \cdot \mathrm{h} / \mathrm{m}^{2}\right)$ calculados en HULC, obteniéndose el coste óptimo representado en las Figuras 9 y 10. Aquí es el edificio entre medianeras el que ofrece menores consumos.

Los resultados permiten seleccionar el coste óptimo más adecuado entre las diferentes propuestas, de forma que los valores del eje $\mathrm{x}$ indican el nivel óptimo de rentabilidad; $\mathrm{y}$ para aquellas propuestas con costes similares, la que tenga el menor uso de energía primaria será la que defina el nivel óptimo.
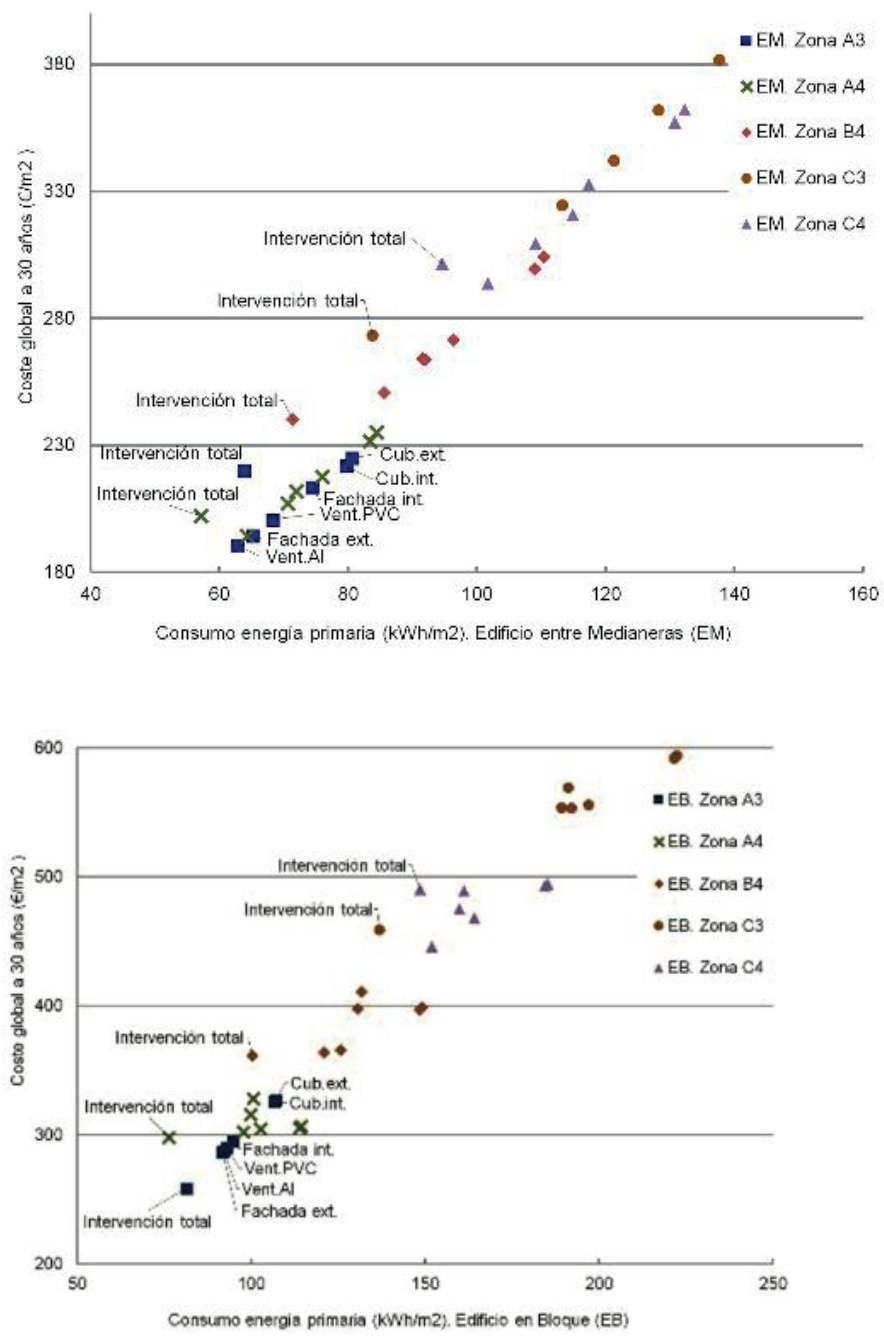

Figura 9: Coste óptimo. Edificio medianeras (EM). Fuente: Elaboración del autor.

Figura 10. Coste óptimo. Edificio bloque (EB).Fuente: Elaboración del autor. 


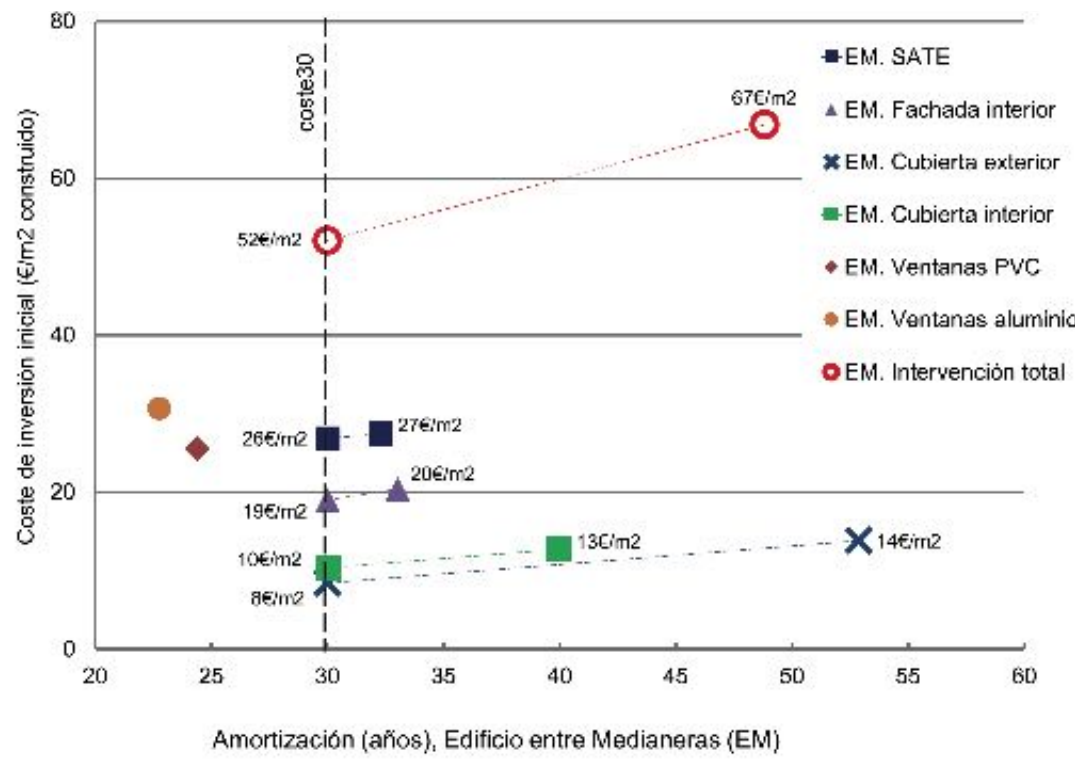

Figura 11. Amortizaciones (años) y "coste30" (€/m2), valores medios de las 5 zonas climáticas. Edificio medianeras (EM). Fuente: Elaboración del autor.

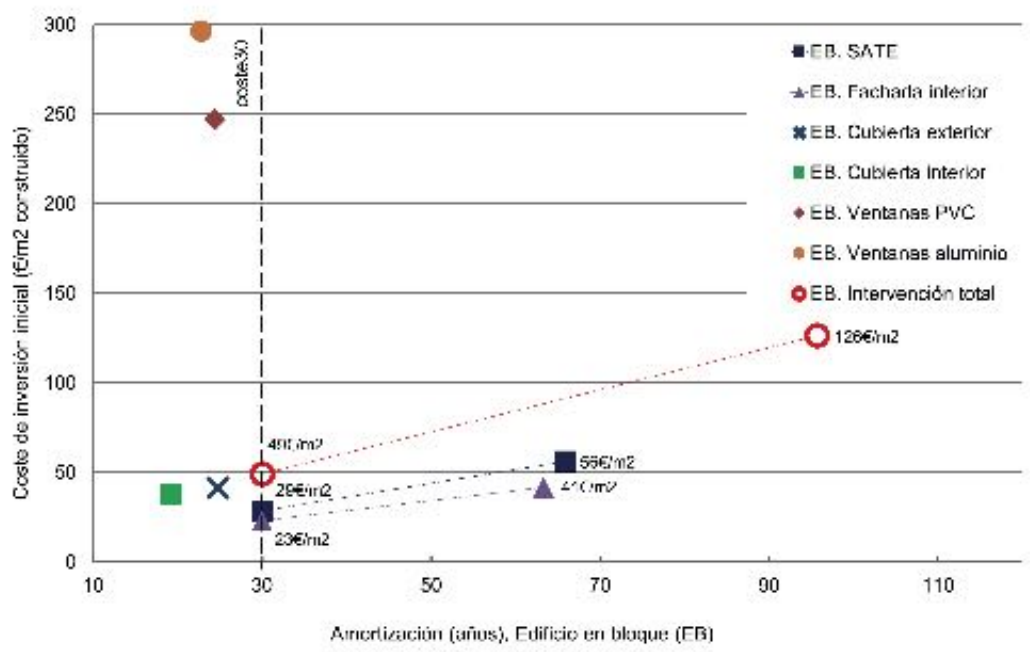

Figura 12. Amortizaciones (años) y "coste30" (€/m2), valores medios de las 5 zonas climáticas. Edificio Bloque (EB). Fuente: Elaboración del autor.

\section{AMORTIZACIONES Y PROPUESTA DE "COSTE30"}

Una vez determinado el coste óptimo se calculan los períodos de amortización (años) de cada intervención, dividiendo el coste de inversión inicial (€) entre el ahorro de energía obtenido (€/año).

Para amortizaciones superiores a 30 años, el estudio propone el valor de "coste30", fijando el valor de amortización y calculando el coste inicial. Este valor es un indicador de cuánto sería necesario abaratar el coste inicial con el fin de obtener amortizaciones en un máximo de 30 años.

En aras de facilitar la interpretación de resultados, todos los valores se han calculado con los valores medios de las cinco zonas climáticas. Las figuras 11 y 12 reflejan las amortizaciones calculadas y los valores de "coste30".

Es necesario indicar que algunas de las medidas ya ofrecen amortizaciones inferiores a 30 años, como las ventanas de PVC o aluminio, en ambos modelos, y las intervenciones de cubierta para edificio en bloque.

Los resultados indican que, para el caso de la intervención total, se requiere reducir el coste de 67 a $52 € / \mathrm{m}^{2}$ construido, en el edificio medianeras, y de 126 a $49 € / \mathrm{m}^{2}$, en el edificio en bloque. 


\section{RESULTADOS Y DISCUSIÓN}

La metodología del coste óptimo se fundamenta en modelos de cálculo energéticos en condiciones de uso estándar, que no dejan de ser estimaciones del comportamiento real de los edificios.

Los resultados de demandas energéticas muestran una gran variabilidad dependiendo de la zona climática: los menores valores se dan en la zona A3 (Cádiz-Málaga) y los mayores, en la zona C3 (Granada).

Es necesario desasociar las amortizaciones con el consumo, ya que consumos elevados ofrecen retornos de la inversión muy bajos. Entre los modelos calculados, el resultado varía notablemente si se consideran perfiles de consumo estándar o altos, cuando las directrices europeas apuestan claramente por reducciones de los consumos energéticos.

Las mejores amortizaciones se obtienen con la renovación de carpinterías, en torno a 23 años, similar a las intervenciones en cubiertas para el caso del edificio en bloque (Figuras 11 y 12).

Los resultados del indicador "coste 30 " ofrecen valores diferentes, según el modelo. En el edificio en bloque, los costes debieran de reducirse un $61 \%$ para la intervención total, y en torno al $45 \%$ para intervenciones en fachada por el exterior e interior. Para el caso del edificio entre medianeras, los resultados son menos exigentes, debido a la menor superficie de envolvente exterior, necesitándose reducciones del 23\% para la intervención total, 35\% en cubiertas y aproximadamente un $5 \%$ en intervenciones en fachada por el exterior e interior.

\section{CONCLUSIONES}

La metodología desarrollada en este trabajo se basa en el marco europeo y permite generar indicadores válidos, sin embargo, la definición de una metodología para España aclararía algunos criterios del cálculo y de precios de la energía que afectan a la obtención de resultados.

Se ha considerado un incremento moderado del $4 \%$ del precio de la energía, pero su variabilidad en un horizonte a 30 años modificaría notablemente los resultados obtenidos; con todo, su encarecimiento mejoraría los plazos de amortización.

No existe un único valor de coste óptimo, dado que se presentan diversas opciones, dependiendo del caso. En la mayoría de ellas, la intervención total proporciona los mejores valores; aunque supone el mayor coste de inversión inicial, este se compensa con la reducción en consumos y costes de energía durante su vida útil de 30 años. También evidencian unos costes óptimos adecuados las intervenciones en fachadas y ventanas.
Considerando las zonas climáticas, los mejores costes óptimos se obtienen para las zonas A3 y A4, representativas de climas más suaves y con menor gastos en consumos. Respecto a los modelos, dichos costes resultan para el edificio entre medianeras, que es representativo de inmuebles de baja calidad constructiva y que ofrece un amplio margen de mejora en la reducción de consumos energéticos.

Además del coste óptimo, es necesario incluir en la contabilidad energética el parámetro de renta familiar y valorar las intervenciones en base a los ingresos salariales. En ese sentido, la variante de intervención total es la que supone más esfuerzo económico, siendo más adecuadas, por su menor coste inicial, las mejoras en fachadas y ventanas. Existen diferentes niveles de inversión, que abarcan desde los $440 € /$ familia hasta los $17.960 € /$ familia, entre las que se plantea un abanico de propuestas que suponen desde 1 a 26 veces el salario mínimo interprofesional.

En relación a las amortizaciones, no todas lasintervenciones son viables y dependen del tipo de edificio y de la zona climática. Por ello, el estudio plantea el indicador "coste30" como un valor adecuado para fijar costes para amortizaciones a 30 años. En el caso del edificio entre medianeras, no es necesario reducir significativamente el coste de inversión, por el contrario, en el del edificio en bloque, algunas de las soluciones debieran de reducir más del $60 \%$ de sus costes. Esta medida se podría incentivar con planes de ayudas y subvenciones estatales, o bien, a través del abaratamiento de los productos.

\section{REFERENCIAS BIBLIOGRÁFICAS}

AENOR-CEN (2008). UNE-EN 15459:2008 Eficiencia energética de los edificios. Procedimiento de evaluación económica de los sistemas energéticos de los edificios. Recuperado de https:// www.une.org.

Arumägi, E., Simson, R., Kuusk, K., Kalamees, T. y Kurnitski, J. (2017). Analysis of cost-optimal minimum energy efficiency requirements for buildings. Brussels: Tallinn University of Technology-European Union.

ATAYO (s/f). Base de Precios PREOC. Recuperado de http:// www.preoc.es/

CA-EPBD. Concerted Action Energy Performance of Buildings Directive (2016). Implementing the Energy Performance of Buildings Directive (EPBD). Brussels: Adene.

COAATGU (s/f). Base de Precios Centro. Recuperado de http:// www.preciocentro.com/

Código Técnico de la Edificación - CTE (2017). Documento Básico Habitabilidad Energía 1. Limitación de la demanda energética: junio 2017. España: Ministerio de Fomento. 
Consejería de Medio Ambiente, Junta de Andalucía (2019). Caracterización Climática de Andalucía, Regiones Climáticas de Andalucía. Recuperado de http://www.juntadeandalucia.es/ medioambiente.

Corrado, V., Ballarini, I. y Paduos, S. (2014). Assessment of Cost-optimal Energy Performance Requirements for the Italian Residential Building Stock. Energy and Buildings, (45), 443-452. DOI: https://doi.org/10.1016/j.egypro.2014.01.048.

Danosa (s/f). Tarifa general. Recuperado de https://portal.danosa. com/

De la Cruz, S., De la Cruz, L. y Simón, J. (2018). Estudio del óptimo económico en rehabilitación de viviendas sociales en función del binomio aislamiento-ahorro de energía. En: CONTART (Zaragoza, 30 mayo -1 junio de 2018): VII Convención de la Edificación (pp. 665-675). Zaragoza: Escuela Universitaria Politécnica de La Almunia.

ECOFYS y European Insulation Manufacturers AssociationEURIMA (2012). Renovation tracks for Europe up to 2050. Building renovation in Europe, what are the choices? Berlín: ECOFYS.

ECOFYS y European Insulation Manufacturers AssociationEURIMA (2015). Assessment of cost optimal calculations in the context of the EPBD (ENER/C3/2013-414), final report. Berlín: ECOFYS

Episcope Project (2016). Energy Performance Indicator Tracking Schemes for the Continuous Optimisation of Refurbishment Processes in European Housing Stocks. Bruselles: Unión Europea. Recuperado de http://episcope.eu/welcome/

European Commission (2007). Passive-on Project. The passivehaus standard in European warm climates. Design guidelines for comfortable energy homes, Part 3. Comfort, climate and passive strategies. Nottingham: Brian Ford.

García, P. y Croxford, B. (2015). Policies to reduce residential energy consumption in Región Metropolitana of Chile, by socioeconomic status and home. Hábitat Sustentable, 2(2), 2-18.

Gobierno de España. Factores de emisión de $\mathrm{CO}^{2}$ y coeficientes de paso a energía primaria de diferentes fuentes de energía final consumidas en el sector de edificios en España. Madrid: Ministerio de Industria, Energía y Turismo - Ministerio de Fomento, 2016.

Herramienta Unificada LIDER-CALENER [programa informático]HULC (2017). Madrid: Ministerio de Vivienda de España, IDAE.

Instituto Nacional Estadística - INE (2019). Censos de Población y viviendas. Recuperado de http://www.ine.es.

Instituto para la Diversificación y Ahorro de la Energía - IDAE. Informe de precios energéticos regulados $n^{\circ} 25$. Madrid: IDAE, 2016.

Junta de Andalucía (2017). Base Costes Construcción Andalucía (BCCA). Recuperado de https://www.juntadeandalucia.es/ organismos/fomentoinfraestructurasyordenaciondelterritorio/ areas/vivienda-rehabilitacion/planes-instrumentos/paginas/bccasept-2017.html
Luxán, M. (2017). Re-habilitación exprés para hogares vulnerables. Soluciones de bajo coste. $1^{\text {a }}$ ed. Madrid: Fundación Gas Natural Fenosa.

Mercado, M., Esteves, A., Barea, G. y Filippín, C. (2018). Efecto de la ventilación natural en el consumo energético de un edificio bioclimático. Análisis y estudio mediante Energy Plus. Revista Hábitat Sustentable, 8(1), 55-67. DOI: http://dx.doi.org/10.22320 /07190700.2018.08.01.05.

Ministerio de Fomento (2013). Report on cost optimal calculations and comparison with the current and future energy performance requirements of buildings in Spain. Madrid: Ministry of Development of Spain.

Ministerio de Fomento (2018). Construcción de edificios (licencias municipales de obra). Años 2013 - 2017. Recuperado de https://www.fomento.gob.es/recursos_mfom/listado/recursos/ ce2013_2017.pdf

Molina, M. y Fernández-Ans, P. (2013). Evolución del comportamiento térmico en viviendas tradicionales de piedra y cubierta de paja. Puesta en valor de un modelo sostenible para el noroeste de España. Revista de la Construcción, 12, 58-67. DOI: http://dx.doi.org/10.4067/S0718-915X2013000200008.

Niemelä, T., Kosonen, R. y Jokisalo, J. (2016). Cost-optimal energy performance renovation measures of educational buildings in cold climate. Applied Energy, (183), 1005-1020. DOI: http:// dx.doi.org/10.1016/j.apenergy.2016.09.044.

Pérez, A., Calama, J.M. y Flores, V. (2016). Comparativa de resultados de rehabilitación energética para viviendas en función del grado de mejora. Informes de la Construcción, 68(541), e134. DOI: http://dx.doi.org/10.3989/ic.15.048.

Re-Programa (2015). (Re)habitación + (Re)generación + (Re) programación. Sevilla: Consejería de Fomento y Vivienda, Junta de Andalucía.

Suárez, R. y Fragoso, J. (2016). Estrategias pasivas de optimización energética de la vivienda social en clima mediterráneo. Informes de la Construcción, 68(541), e136. DOI: https://doi.org/10.3989/ ic.15.050.

TABULA Project (2012). Typology Approach for Building Stock Energy Assessment, 2009-2012. Executive Summary. Bruselles: Unión Europea.

Unión Europea (2010). Directiva 2010/31/EU del Parlamento Europeo y del Consejo, relativa a la eficiencia energética de los edificios (refundición). Diario Oficial de la Unión Europea, $n^{\circ} \mathrm{L}$ 153.

Unión Europea (2012a). Reglamento Delegado RD 244/2012 de la Comisión de 16 de enero de 2012 que complementa la Directiva 2010/31/UE. Diario Oficial de la Unión Europea, n L 81.

Unión Europea (2012b). Directrices que acompañan al Reglamento Delegado RD 244/2012. Diario Oficial de la Unión Europea, 2012/C 115/01. 
Unión Europea (2019). Driving energy efficiency in the European building stock: New recommendations on the modernisation of buildings. Recuperado de https://ec.europa.eu/info/news/ driving-energy-efficiency-european-building-stock-newrecommendations-modernisation-buildings-2019-jun-21_en.

URSA (s/f). Lista de precios recomendados. Recuperado de https://www.ursa.es/

Zangheri, P., Armani, R., Pietrobon, M. y Pagliano, L. (2018). Identification of cost-optimal and NZEB refurbishment levels for representative climates and building typologies across Europe. Energy Efficiency, 11, 337-369. DOI: https://doi.org/10.1007/ s12053-017-9566-8. 